\title{
Pattern and Final Visual Outcome in Paediatric Ocular Trauma in Central India
}

\author{
Bharti Badlani ${ }^{1}$, Dharmendra Singh Saryyam ${ }^{2}$, Anil Kumar Raichoor ${ }^{3}$, Manisha Bhatt Dwivedi ${ }^{4}$ \\ ${ }^{1}$ Department of Ophthalmology, CIMS, Chhindwara, Madhya Pradesh, India. ${ }^{2}$ Department of \\ Ophthalmology, SSIMS, Bhilai, Chhattisgarh, India. ${ }^{3}$ Department of Dentistry, CIMS, Chhindwara, \\ Madhya Pradesh, India. ${ }^{4}$ Department of Anesthesia, SSIMS, Bhilai, Chhattisgarh, India.
}

\section{ABSTRACT}

\section{BACKGROUND}

Paediatric ocular trauma is one of the most common causes of preventable visual morbidity in children. One third vision loss occurs in the first decade of life, which is crucial stage for visual development. We wanted to evaluate the final visual outcome of paediatric ocular trauma and determine the various aetiological factors contributing to ocular trauma.

\section{METHODS}

Prospective data regarding age, sex, visual acuity, etiological factors of 100 children who visited the emergency in the department of ophthalmology was collected. Children below 18 years of age were included. Children who could not cooperate or lost to follow-up were excluded from the study. Chi square test was done. P values were considered statistically significant at $0.01 \%$ level.

\section{RESULTS}

Of the 100 cases, closed globe injury accounted for $69 \%$ and the sex ratio was 3.2:1. 48 children had visual acuity $>6 / 12,18$ had visual acuity $6 / 18-6 / 36$, and 3 children had no perception of light (NOPL).

\section{CONCLUSIONS}

Paediatric ocular trauma is a major cause of the non-congenital unilateral blindness. Such injuries cannot always be prevented, but by identifying the underlying aetiological factor of serious injuries, it may be possible to determine the most effective method of reducing the incidence of visually challenging trauma. Although prognosis is mainly dependent on the extent of injury, choice of appropriate management can favourably affect the visual outcome.

\section{KEY WORDS}

Paediatric Ocular Trauma, Visual Acuity, Closed Globe Injuries, Open Globe Injuries, Final Visual Outcome
Corresponding Author: Dr. Anil Kumar Raichoor, H. No. A-31, Arcons City, Khajri, Chhindwara, Madhya Pradesh, India. E-mail:drrakumar@yahoo.com

DOI: $10.14260 /$ jemds/2020/361

Financial or Other Competing Interests: None.

How to Cite This Article:

Badlani B, Saryyam DS, Raichoor AK, et al. Pattern and final visual outcome in paediatric ocular trauma in Central India. J. Evolution Med. Dent. Sci. 2020;9(21):16461648, DOI: $10.14260 /$ jemds/2020/361

Submission 25-01-2020, Peer Review 05-05-2020

Acceptance 11-05-2020,

Published 25-05-2020.

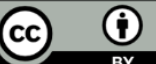




\section{BACKGROUND}

It is estimated that there are up to 2,80,000 hospital admission due to ocular trauma in children less than 15 years of age each year. ${ }^{1}$ Of the total childhood injuries ocular trauma accounts for about $8-14 \%{ }^{2}$ which is also the leading cause of treatable visual morbidity and preventable monocular blindness. The ensuing visual disability has significant, socioeconomic and psychological impact on the children, family and to the society. ${ }^{3}$ Which warrants accurate estimate of the prevalence and the aetiological factors contributing to the ocular trauma and will be helpful in preventing as well as better management and cure of the patients. There are very limited epidemiological studies in India, or none in central India. This study will help in estimating the contributing factors responsible for preventable blindness in children.

Prevention of severe and blinding ocular injuries by better educational efforts, parental supervision during sporting activities, domestic safety measures and early medical intervention are important decisive factors in decreasing the morbidity and incidence of ocular injuries. In view of various complexities involved this study would provide an efficient multi-dimensional approach towards the management of paediatric ocular trauma.

We wanted to evaluate the final visual outcome of paediatric ocular trauma and determine the various types of aetiological factors contributing to ocular trauma.

\section{METHODS}

After obtaining approval from the institutional ethics committee, a prospective study was done among children who reported with ocular trauma to the department of ophthalmology. 100 children with ocular trauma who were less than $18 \mathrm{y}$ of age were included in this study. Uncooperative children and who could not attend the follow up were excluded. Classification of Pieramici et al was adapted in this study. Clinical assessment of every patient was done by obtaining Visual acuity, age, sex, duration from time of injury to reporting in the hospital, place of injury, aetiology of injury and local examination. Ocular examination best corrected Pretreatment visual acuity with Snellen chart or multiple picture test in verbal children, whereas in preverbal children. Estimation of visual acuity was done by fixing and following the light or by hundred and thousand sweet tests.

In all cases anterior segment was examined by oblique illumination with torch light using binocular loupe and slit lamp. Fundus examination with direct and indirect ophthalmoscope was done for evidence of intra ocular foreign body, vitreous haemorrhage and opacities, oedema or detachment of retina and condition of optic disc. Fundus examination was not possible in patients with massive hyphaema and massive vitreous haemorrhage.

In some cases, ultra-sonography and x-ray of the orbit was done to rule out intra ocular foreign body (IOFB) or orbital fracture. Depending on the type of injury patients were either managed by conservative or surgical methods. 57 patients were managed conservatively, and 43 patients required surgical intervention. Conservative treatment was given in cases of closed globe injury which included lid oedema, ecchymosis, conjunctival congestion / chemosis, subconjunctival haemorrhage, corneal abrasion / partial thickness tear, traumatic uveitis, hyphaema $<1 / 2$ of anterior chamber, traumatic mydriasis, Iridodialysis $(<2 \mathrm{hrs}$. meridian asymptomatic), vitreas haemorrhage, Berlin's oedema and retinal haemorrhage.

Surgery was required for lid tear, conjunctival tear, foreign body, corneal tear with or without Iris / vitreous prolapses, traumatic cataract, subluxation or dislocation of lens and endophthalmitis. All the patients were followed up at intervals of 1, 2, 4 weeks and 3, 6 months. In every follow up visual acuity, anterior and posterior segment examination was done. Tonometry if required in certain patients was also done.

\section{Statistical Analysis}

Chi square test was used and $\mathrm{P}$ value at $0.01 \%$ level was considered to be statistically significant.

\section{RESULTS}

In this study higher incidence of ocular trauma was seen in the 5-14 years age group which accounted for $66 \%$ of the total cases. The sex ratio between male and female child was 3.2:1 (table-1). The most common aetiological factor was injury by wooden stick (14.5\%), however interpersonal fights, injury due to iron nails and thick rope were least common (2.9\%) of total cases (table-2). Closed globe injury was the most common type of injury reported in this study accounting for $69 \%$ and burns accounting for $8 \%$ of the total injuries (table $3)$.

\begin{tabular}{|cccccc|}
\hline Age Group & \multicolumn{3}{c}{ Male } & \multicolumn{2}{c|}{ Female } \\
(Yrs.) & Number & Percentage & Number & Percentage & Ratio \\
$0-5$ & 13 & $17.1 \%$ & 04 & $16.7 \%$ & $3.3: 1$ \\
$5-14$ & 50 & $65.8 \%$ & 16 & $66.6 \%$ & $3.1: 1$ \\
$14-18$ & 13 & $17.1 \%$ & 04 & $16.7 \%$ & $3.3: 1$ \\
Total & $\mathbf{7 6}$ & $\mathbf{2 4}$ & $\mathbf{3 . 2 : 1}$ \\
\hline \multicolumn{5}{c}{ Table 1. Demographic Profile of Patients } \\
\hline
\end{tabular}

\begin{tabular}{|ccc|}
\hline Etiological Factors (n=69) & Number & Percentage \\
Wooden stick & 10 & $14.5 \%$ \\
Cricket Ball & 09 & $13.0 \%$ \\
Gulli danda & 09 & $13.0 \%$ \\
Fall & 08 & $11.6 \%$ \\
Stone/Gravel & 08 & $11.6 \%$ \\
Toys & 05 & $7.2 \%$ \\
Tree Twig & 05 & $7.2 \%$ \\
Fist & 04 & $5.8 \%$ \\
Iron Nail & 02 & $2.9 \%$ \\
Slap & 02 & $2.9 \%$ \\
Thick rope & 02 & $2.9 \%$ \\
Unknown & 05 & $7.2 \%$ \\
Total & $\mathbf{6 9}$ \\
\hline \multicolumn{2}{c}{ Table 2. Etiological Factors } \\
\hline
\end{tabular}

Of the 100 patients 48 had visual acuity (pre-treatment) > $6 / 12,18$ patients have visual acuity 6/18-6/36 and 3 cases had NOPL (table-4). In the final visual outcome $63 \%$ patients had good visual acuity $>6 / 12,23 \%$ had visual acuity $6 / 18$ $6 / 36.9 \%$ patients had visual acuity $6 / 60-2 / 60$ and $2 \%$ cases has visual acuity $1 / 60$ to PL. $3 \%$ cases had NOPL. The comparison of pre-treatment visual outcome was statistically significant at $0.01 \%$ level. 


\begin{tabular}{|ccc|}
\hline Type of Injury & Number & Percentage \\
Close Globe & 69 & $69.0 \%$ \\
Open Globe & 23 & $23.0 \%$ \\
Burn & 08 & $08.0 \%$ \\
Total & $\mathbf{1 0 0}$ & $\mathbf{1 0 0 . 0 \%}$ \\
\hline \multicolumn{3}{c}{ Table 3. Type of Injury } \\
\hline
\end{tabular}

\begin{tabular}{|ccc|}
\hline Visual Acuity & Number & Percentage \\
$>6 / 12$ & 48 & $48.0 \%$ \\
$6 / 18-6 / 36$ & 18 & $18.0 \%$ \\
$6 / 60-2 / 60$ & 21 & $21.0 \%$ \\
1/60-PL & 10 & $10.0 \%$ \\
NOPL & 03 & $03.0 \%$ \\
Total & $\mathbf{1 0 0}$ & $\mathbf{1 0 0 . 0} \%$ \\
\hline \multicolumn{3}{c}{ Table 4. Pre-Treatment Visual Acuity } \\
\hline
\end{tabular}

\section{DISCUSSION}

This study was motivated by the fact that there is lack of research data concerning the prevalence of final visual outcome in paediatric ocular trauma in central India. Ocular trauma constitutes about $12.9 \%$ of all cases admitted in developing countries. In our study it was found that the most common age group involved was 5-14 years similar to other studies by Puodziuvlene et al (older than 7 years), ${ }^{4}$ and Chakraborti $\mathrm{C}$ et al ${ }^{5}$ respectively. However male children were more prone to ocular injuries with a ratio of 3.2:1 which is consistent with various other studies, this could be due to the fact that boys tend to take part in more violent and aggressive nature of activities than girl's in this age group.

Injury due to wooden stick $14.5 \%$ was the most common aetiological factor in our study similar to in a study by Puodziuvlene et al who also reported $24.8 \%$ wooden stick injuries, also children are routinely engaged in farming or domestic chores in as many household lack proper intra structure especially in rural India. According to 2009-10 census there was a gross dropout rate of $26.3 \%$ in (class I to VIII) out of which one fourth children were employed in agriculture sector.

Close globe injury accounted for almost $69 \%$ of the total type of injuries in our study which could be due to the fact that they were sustained while playing in school.

Concerning the final visual outcome in our study $63 \%$ patients had good visual acuity $>6 / 12$, however Narang et al ${ }^{6}$ reported ambulatory vision in $52.8 \%$ of children in their study and Puodziuvlene et al reported $65.4 \%$ regained a good visual acuity $(>0.5)$ the reason for the better outcome of final visual acuity in our study could be due to the fact that majority of the patients sustained closed globe injuries similar to in a study by
Katiyar. V et al.7 Studies on paediatric trauma have reported poor visual acuity at presentation along with open globe injuries among the predictors associated with poor visual outcome. In our study $3 \%$ of the patient had monocular blindness (NOPL) as compared to Shazia Qayum et al ${ }^{8}$ who reported almost $12.3 \%$ in their study, which can be attributed to greater percentage of open globe injuries in their study.

\section{CONCLUSIONS}

By identifying the underlying etiological factor and determining the appropriate management, good visual acuity can be achieved in children with closed globe injuries who present early irrespective of the type of injury.

\section{REFERENCES}

[1] Brophy M, Sinclair SA, Hostetlier SG, et al. Pediatric eye injury-related hospitalizations in the United States. Pediatrics 2006;117:1263-71.

[2] Nelson LB, Wilson TW, Jeffers JB. Eye injuries in childhood: demography, etiology and prevention. Pediatrics 1989;84(3):438-41.

[3] Saxena R, Sinha R, Purohit A, et al. Pattern of paediatric ocular trauma in India. Indian J Pediatr 2002;69(10):8637.

[4] Puodziuvlene E, Jokubauskiene G, Vieversyte M, et al. A five year retrospective study of the epidemiological characteristics and visual outcomes of pediatric ocular trauma. BMC Ophthalmology 2018;18(1):10.

[5] Chakraborti C, Giri D, Choudhury KP, et al. Paediatric ocular trauma in a tertiary eye care centre in Eastern India. Indian J Public Health 2014;58(4):278-80.

[6] Narang S, Gupta V, Simalandhi P, et al. Paediatric open globe injuries. Visual outcome and risk factors for endophthalmitis. Indian J Ophthalmol 2004;52(1):29-34.

[7] Katiyar V, Bangwal S, Gupta SK, et al. Ocular trauma in Indian pediatric population. J Clin Ophthalmol Res 2016;4(1):19-23.

[8] Shazia Q, Rashid A, Shagufta R. Epidemiological profile of pediatric ocular trauma in a tertiary hospital of northern India. Chin J Traumatol 2018;21(2):100-03. 